Center for

Mathematical Economics

Working Papers

April 2016

\title{
On Preemption in Discrete and Continuous Time
}

Jan-Henrik Steg

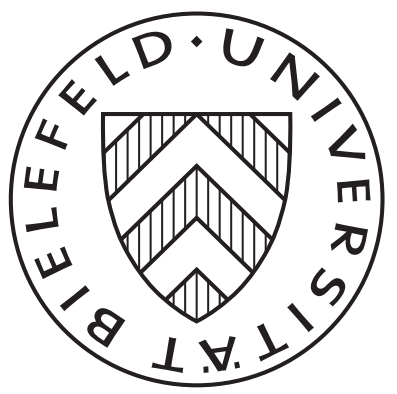

Center for Mathematical Economics (IMW) 


\title{
On Preemption in Discrete and Continuous Time
}

\author{
Jan-Henrik Steg*
}

\begin{abstract}
The seminal work of Fudenberg and Tirole (1985) on how preemption erodes the value of an option to wait raises general questions about the relation between models in discrete and continuous time and thus about the interpretation of its central result, relying on an "infinitely fine grid". Here it is shown that the preemption equilibrium is the limit of the unique symmetric equilibria of the game when reduced to any sequence of grids becoming infinitely fine. Furthermore, additional subgame perfect equilibria using conventional continuous-time mixed strategies are identified.
\end{abstract}

Keywords: Preemption, discrete time, continuous time, subgame perfect equilibrium, convergence.

JEL subject classification: C61, C73, D21, D43, L12, L13

\section{Preemption in discrete and continuous time}

Preemption is a well-known phenomenon in the context of irreversible investment. In their seminal paper, Fudenberg and Tirole (1985, henceforth FT) argue forcefully that a first-mover advantage provides an incentive for two firms to preempt each other in adopting a new technology, such that, in equilibrium, adoption occurs so early that the firms are indifferent between being the first adopter (called leader) or the second (called follower). Thus competition diminishes the value of the option to wait for an optimal adoption time. ${ }^{1}$ This reasoning has a drastic influence on the valuation of real options in general and has been applied extensively in the literature. ${ }^{2}$

\footnotetext{
${ }^{*}$ Center for Mathematical Economics, Bielefeld University, Germany. jsteg@uni-bielefeld.de Financial support by the German Research Foundation (DFG) via grant Ri 1142-4-2 is gratefully acknowledged.

${ }^{1}$ This effect requires to model subgame perfect reactions to any observed adoption by some firm, and thus to distinguish histories in which some firm already has adopted or not, respectively. As it is customary in timing games, FT fix optimal reactions and concentrate on the game for histories in which no firm has adopted. Reinganum (1981), on the contrary, studies simple Nash equilibria in which the firms precommit to adoption times and thus, if preempted, do not postpone adoption as would be optimal.

${ }^{2}$ See, e.g., Grenadier (1996) or Weeds (2002), or, for a recent survey on game-theoretic real option models, Azevedo and Paxson (2014).
} 
However, FT make their argument by means of a continuous-time model using unconventional mixed strategies, which are motivated by discrete-time limits, but lack a formal link to discrete-time versions of the game. ${ }^{3}$ That link will be established here. In general, the interest in the relation between limits of discrete-time games and games framed in continuous time, which has recently been investigated for different classes of games, ${ }^{4}$ is due to the following dilemma. A continuous-time model may be desirable by the promise to apply convenient analytic methods, or because it seems unnatural to restrict the timing of actions in a given problem. However, one often encounters conceptual problems to define reasonable strategies in continuous time, or equilibrium existence may be lost, which is typical for timing games.

A general modeling issue for timing games is the flip side of the intuitive preemption argument: which strategies can actually support an equilibrium of early stopping at the point where the firms are indifferent between the leader and follower roles. A firm is only willing to adopt at that point if any hesitation would result in being preempted, because the payoff to the leader $(L)$ is increasing at that point, see time $T_{1}$ in Figure 1 . There cannot be simultaneous adoption, which is the worst outcome $(M)$. Thus, the respective other firm
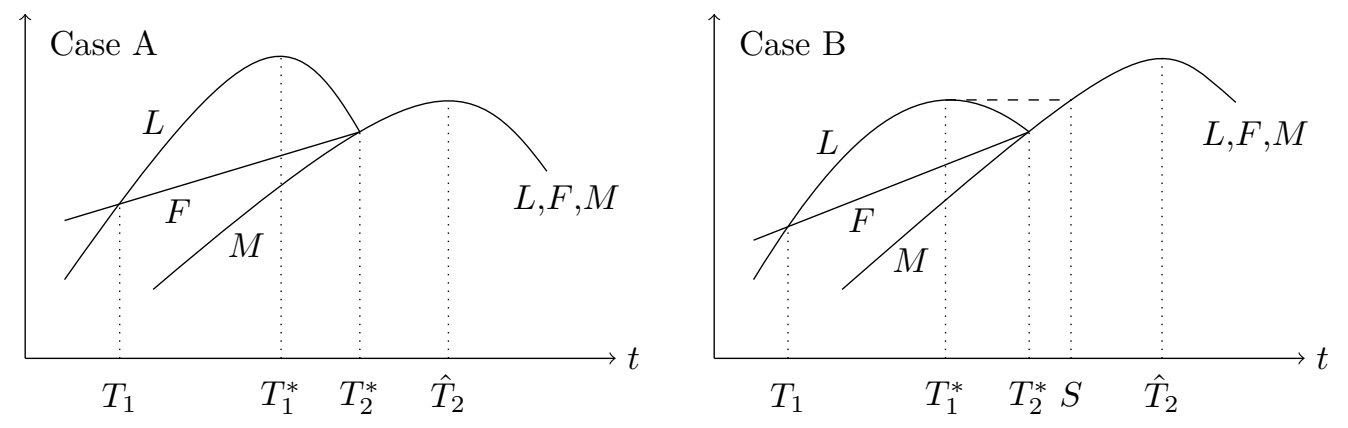

Figure 1: Values at first adoption time $t$.

must use a strategy that induces adoption "immediately after" the indifference point $T_{1}$ if no adoption has occured at $T_{1}$ (or before), which in continuous time can only mean adopting at a rate on an interval from $T_{1}$. That rate would have to be infinite if the associated probability of getting the follower payoff $(F)$ was to compensate the increase in $L$, so there is no preemption equilibrium in conventional distributions over time. ${ }^{5}$

FT let the firms instead place "atoms" $\alpha(t) \in(0,1)$ on every $t \in\left(T_{1}, T_{2}^{*}\right)$ and interpret

\footnotetext{
${ }^{3}$ Simon and Stinchcombe (1989) formalize the idea of an "infinitely fine grid" for extensive-form games with pure strategies. Fudenberg and Levine (1986) consider continuous-time limits of $\varepsilon$-Nash equilibria of discretetime versions of (timing) games. Laraki et al. (2005) construct $\varepsilon$-subgame perfect equilibria for continuous time games of timing by discretizing time, but without taking a limit.

${ }^{4}$ See, e.g., Fudenberg and Levine (2009) and the references therein.

${ }^{5}$ To make a firm willing to adopt, the other has to adopt at least with the hazard rate $L^{\prime}(t) /[L(t)-F(t)]$ from $T_{1}$ on, see Hendricks and Wilson (1992). This rate is not integrable for the model of FT and would thus result in a unit mass on $\left\{T_{1}\right\}$, see Lemma 5.1 in Section 5 .
} 
these as conditional adoption probabilities. Requiring the atoms to be a continuous function of time, the outcome at $t$ is defined to be that from playing an infinite sequence of constant probabilities $\alpha(t)$. The analogy with discrete-time models is only illustrated by a simpler example (a "grab-the-dollar" coordination game with monotone payoffs). As equilibria of games in discrete time are very sensitive to the last period (like the prisoners' dilemma or the chain store paradox), the relation of their proposed solution to limits of actual discrete-time versions of the game is not clear.

In Section 3 we show that there is a unique symmetric subgame perfect equilibrium if the adoption times are confined to any discrete grid (Proposition 3.1). For any sequence of grids that become arbitrarily fine, the equilibrium distribution over outcomes does converge to the preemption equilibrium outcome distribution proposed by FT (in any subgame, Theorem 3.2). In particular, the equilibrium payoffs in any subgame with $L(t) \geq F(t)$ converge to $F(t)$ and immediate adoption by at least one firm occurs, whereas the payoffs in any subgame with $L(t)<F(t)$ converge to $L\left(T_{1}\right)$, where the first adoption occurs, with probability $1 / 2$ by either firm. Concerning the strategies, for any fixed $t \in \mathbb{R}_{+}$and $l \in \mathbb{N}$, the adoption probabilities in the first $l$ periods after $t$ converge uniformly to $\alpha(t)$ and thus the limit interpretation of FT is valid. In particular, for any $t \in\left(T_{1}, T_{2}^{*}\right)$ the limit is

$$
\alpha(t)=\frac{L(t)-F(t)}{L(t)-M(t)} \in(0,1)
$$

and by hesitating for a positive amount of time, any firm would be preempted immediately in the limit and become follower. As waiting must also be optimal given the fully mixed strategies, the payoff must be that of the follower.

The analysis leading to this result is based on the dynamics of the equilibrium continuation values that are implied by the indifference condition from playing a mixed strategy in any equilibrium. A general key insight from this analysis is that the continuation values are nondecreasing in time where $F>M$ (Lemma 5.2). Further, where $L>F$, the limit of the continuation values at time $t$ cannot lie in $(F, L)$ (Lemma 5.4), so the limit must be $F$.

In discrete time there are additional equilibria in which the firms take turns of adopting in periods with a first-mover advantage. Whether there is a limit outcome at some $t$ (even in payoffs, for $\left.t \in\left(T_{1}, T_{2}^{*}\right)\right)$ depends strongly on the sequence of grids and on who starts with adopting on any grid. There are also continuous-time equilibria that are not limits from discrete time. FT show that preemption can be avoided in Case B in Figure 1 by firms agreeing on any joint adoption date in $\left[S, \hat{T}_{2}\right]{ }^{6}$ These are equilibria in pure strategies.

Although FT allow the firms to use conventional continuous-time mixed strategies - distribution functions $G(t)$ over time - they miss some subgame perfect equilibria using such strategies in their equilibrium classification. In Section 4 below, Theorem 4.1 exhibits equi-

\footnotetext{
${ }^{6}$ Such equilibria could however be obtained as limits of $\varepsilon$-equilibria with $\varepsilon \searrow 0$ as the period length vanishes.
} 
libria using nondegenerate mixed strategies. In these equilibria, the firms also coordinate on late joint adoption after $S$. From some arbitrary earlier point between $T_{1}$ and $S$ on, however, each firm thinks that the other will adopt in the next instant with a certain small probability, and not after $S$. The associated risk of becoming follower balances the increase in $L$ and thus adoption becomes as good as waiting.

This principle holds although $L$ is not monotone. It also does not depend on the specific payoff processes $L, F$ and $M$ here, but works much more generally for other nonmonotone processes. Therefore, the equilibrium verification step for Theorem 4.1 is formulated as a separate Proposition 5.5 with all requirements on $L, F$ and $M$ in its statement, to be found in Section 5 that contains all proofs.

\section{The model}

As in FT, we start with their basic technology adoption model and derive payoffs $L(t), F(t)$ and $M(t)$ that accrue if $t$ is the first time of adoption, on which the subsequent analysis will be based. Consider two firms having the option to adopt some available new technology. The cost of adopting at time $t \in \mathbb{R}_{+}$is $c(t)$ and falling over time. Before adopting, a firm earns the revenue $\pi_{0}(m)$ per unit of time, where $m$ is the number of firms already having adopted; it switches to $\pi_{1}(m)$ by adoption. Revenues are discounted continuously at the rate $r>0$, whereas $c$ is already discounted to $t=0$. Thus, if firms $i, j \in\{1,2\}, i \neq j$ adopt at times $t_{i}, t_{j} \in \mathbb{R}_{+}$, the payoff to firm $i$ is

$$
V\left(t_{i}, t_{j}\right)= \begin{cases}\int_{0}^{t_{i}} e^{-r s} \pi_{0}(0) d s+\int_{t_{i}}^{t_{j}} e^{-r s} \pi_{1}(1) d s+\int_{t_{j}}^{\infty} e^{-r s} \pi_{1}(2) d s-c\left(t_{i}\right) & \text { if } t_{i}<t_{j}, \\ \int_{0}^{t_{j}} e^{-r s} \pi_{0}(0) d s+\int_{t_{j}}^{t_{i}} e^{-r s} \pi_{0}(1) d s+\int_{t_{i}}^{\infty} e^{-r s} \pi_{1}(2) d s-c\left(t_{i}\right) & \text { if } t_{i} \geq t_{j} .\end{cases}
$$

It is assumed that

$$
\pi_{1}(1)-\pi_{0}(0)>\pi_{1}(2)-\pi_{0}(1) \geq \pi_{1}(2)-\pi_{0}(0),
$$

i.e. the gain of the first adopter exceeds that of the second, and adopting does not increase the revenue of the other firm.

If firm $j$ is the only adopter by time $t_{j} \in \mathbb{R}_{+}$, an optimal adoption time for the other firm $i$ maximizes $V\left(t_{i}, t_{j}\right)$ over $t_{i} \geq t_{j}$, which is equivalent to maximizing

$$
\int_{\tau}^{\infty} e^{-r s}\left(\pi_{1}(2)-\pi_{0}(1)\right) d s-c(\tau)
$$

over all feasible times $\tau \geq t$. Assume that (2.2) is strictly quasiconcave in $\tau \in \mathbb{R}_{+}$and has a maximum at some $T_{2}^{*}>0$ (implied by Assumption 2 on $c$ in FT). Thus the unique optimal time to adopt in $\left[t_{j}, \infty\right)$ is $\max \left(T_{2}^{*}, t_{j}\right)$. Therefore, if $t \in \mathbb{R}_{+}$is the first time at which any firm adopts, then the payoff to firm $i$ given an optimal follower reaction in continuous time is 
$L(t):=V\left(t, T_{2}^{*} \vee t\right)$ if firm $i$ is the only first adopter and thus the leader, $F(t):=V\left(T_{2}^{*} \vee t, t\right)$ if firm $j$ is the only first adopter and $i$ the follower, and $M(t):=V(t, t)$ if simultaneous adoption occurs.

Note that $M(t)=F(t)=L(t)$ for all $t \geq T_{2}^{*}$ and $M(t)<\min (F(t), L(t))$ for all $t<T_{2}^{*}$ by uniqueness of the latter and $\pi_{1}(1)>\pi_{1}(2)$, respectively. We will use the following further properties that are stylized in Figure 1 and implied by Assumption 2 on $c$ in FT. $L, F$ and $M$ are continuous. There is a point $T_{1}<T_{2}^{*}$ such that $L(t)<F(t)$ for $t \in\left[0, T_{1}\right)$ and $L(t)>F(t)$ for $t \in\left(T_{1}, T_{2}^{*}\right)$. When restricted to $\left(T_{1}, T_{2}^{*}\right), L$ is strictly quasiconcave and has a maximum at some point $T_{1}^{*}$. Finally, $M$ is strictly quasiconcave and has a maximum at some point $\hat{T}_{2} \geq T_{2}^{*} \cdot{ }^{7}$

\section{Equilibria in discrete time}

Consider a family of grids $\left\{\mathcal{T}^{N} ; N \in \mathbb{N}\right\}$, where each grid is a strictly increasing and unbounded sequence of time points, i.e. $\mathcal{T}^{N}=\left\{t_{0}^{N}, t_{1}^{N}, \ldots\right\}$ with $0=t_{0}^{N}<t_{1}^{N}<\cdots$ and $\sup \mathcal{T}^{N}=\infty$ for every $N \in \mathbb{N}$, but such that its mesh $\left\|\mathcal{T}^{N}\right\|:=\sup \left\{t_{k}^{N}-t_{k-1}^{N} ; k \in \mathbb{N}\right\}$ is finite. Assume that the grids are ordered by decreasing mesh size and that the mesh gets arbitrarily fine, i.e. $\left\|\mathcal{T}^{N}\right\| \searrow 0$ as $N \rightarrow \infty$.

The payoffs at the first time of adoption are as follows if the adoption times are restricted to the grid $\mathcal{T}^{N}$. First, simultaneous adoption at any $t_{k}^{N} \in \mathcal{T}^{N}$ yields payoffs $M^{N}\left(t_{k}^{N}\right):=$ $V\left(t_{k}^{N}, t_{k}^{N}\right)=M\left(t_{k}^{N}\right)$. Now the feasible adoption times for a firm that becomes follower at $t_{k}^{N}$ are $\tau \in \mathcal{T}^{N} \cap\left[t_{k+1}^{N}, \infty\right)$. By strict quasiconcavity, (2.2) is maximized over all $\tau \in \mathcal{T}^{N}$ (only) by some endpoint of the interval $\left[t_{l}^{N}, t_{l+1}^{N}\right)$ that contains $T_{2}^{*}$. Denoting that point by $T_{2}^{* N}$, an optimal adoption time for the follower is $\max \left(T_{2}^{* N}, t_{k+1}^{N}\right)$. Thus, the follower payoff is

$$
F^{N}\left(t_{k}^{N}\right):= \begin{cases}V\left(t_{k+1}^{N}, t_{k}^{N}\right) \leq M^{N}\left(t_{k}^{N}\right) & \text { if } t_{k}^{N} \geq T_{2}^{* N}, \\ V\left(T_{2}^{* N}, t_{k}^{N}\right) \geq M^{N}\left(t_{k}^{N}\right) & \text { if } t_{k}^{N}<T_{2}^{* N},\end{cases}
$$

and the leader payoff is

$$
L^{N}\left(t_{k}^{N}\right):= \begin{cases}V\left(t_{k}^{N}, t_{k+1}^{N}\right) & \text { if } t_{k}^{N} \geq T_{2}^{* N}, \\ V\left(t_{k}^{N}, T_{2}^{* N}\right) & \text { if } t_{k}^{N}<T_{2}^{* N} .\end{cases}
$$

Note that we can have $F^{N}\left(t_{k}^{N}\right)=M^{N}\left(t_{k}^{N}\right)$ for at most one $t_{k}^{N} \in \mathcal{T}^{N}$ by strict quasiconcavity of (2.2), which must be $T_{2}^{* N}$ or its predecessor. Note also that $F^{N}\left(t_{k}^{N}\right) \leq F\left(t_{k}^{N}\right)$ as there are fewer possibilities to optimize on the grid.

\footnotetext{
${ }^{7}$ All quasiconcavity properties hold if $(2.2)$ is also strictly quasiconcave for the other revenue differences from (2.1). Their order implies that of $T_{1}^{*}<T_{2}^{*} \leq \hat{T}_{2}$ if $c$ is differentiable. $T_{1}$ exists if (2.2) is also strictly quasiconcave for $\pi_{1}(1)-\pi_{0}(1)$, as then $L-F$ is strictly quasiconcave on $\left[0, T_{2}^{*}\right]$.
} 
The following properties will be used for the equilibrium determination. Recall that $M(t)$ is strictly quasiconcave and has a maximum at some $\hat{T}_{2} \geq T_{2}^{*}$. Letting $\hat{T}_{2}^{N} \geq T_{2}^{* N}$ denote the right endpoint of the interval $\left[t_{l}^{N}, t_{l+1}^{N}\right)$ containing $\hat{T}_{2}$, also $M^{N}\left(t_{k}^{N}\right)$ is strictly increasing for $t_{k+1}^{N}<\hat{T}_{2}^{N}$ and strictly decreasing for $t_{k}^{N}>\hat{T}_{2}^{N} . L^{N}$, on the contrary, need not resemble $L$ as much due to the follower reaction varying with the period length. A general property is that $L^{N}\left(t_{k}^{N}\right)-M^{N}\left(t_{k}^{N}\right)>0$ for all $t_{k}^{N} \in \mathcal{T}^{N}$, as the difference is the value of the monopoly markup $\pi_{1}(1)-\pi_{1}(2)$ at least over the period $\left[t_{k}^{N}, t_{k+1}^{N}\right)$. By discounting, that difference vanishes as $t_{k}^{N} \rightarrow \infty$.

In the timing game on any grid $\mathcal{T}^{N}$ we focus on the adoption decisions as long as no firm has adopted and determine payoffs at the first time of adoption by the processes $L^{N}, F^{N}$ and $M^{N}$. A behavioral strategy for player $i \in\{1,2\}$ is thus a sequence $\sigma_{i}^{N}: \mathbb{N}_{0} \rightarrow[0,1]$, where $\sigma_{i}^{N}(k)$ is the probability with which firm $i$ adopts at time $t_{k}^{N} \in \mathcal{T}^{N}$ if no firm has adopted before. Given a behavioral strategy for each firm $i$, the strategy profile is denoted $\sigma^{N}=\left(\sigma_{1}^{N}, \sigma_{2}^{N}\right)$ and the associated expected payoff to firm $i$ at time $t_{k}^{N}$ if no firm has adopted, yet, is denoted by $V_{\sigma^{N}}^{i}\left(t_{k}^{N}\right)$.

Given a strategy profile $\sigma^{N}$, firm $i$ is indifferent between adopting and waiting at time $t_{k}^{N}$ if and only if

$$
\left(1-\sigma_{j}^{N}(k)\right) L^{N}\left(t_{k}^{N}\right)+\sigma_{j}^{N}(k) M^{N}\left(t_{k}^{N}\right)=\sigma_{j}^{N}(k) F^{N}\left(t_{k}^{N}\right)+\left(1-\sigma_{j}^{N}(k)\right) V_{\sigma^{N}}^{i}\left(t_{k+1}^{N}\right) .
$$

Adoption is uniquely optimal if the LHS exceeds the RHS, and waiting in the opposite case. The equilibria in Proposition 3.1 are constructed by backward iteration from times $t_{k+1}^{N}>T_{2}^{* N}$ at which adoption is dominant, so $V_{\sigma^{N}}^{i}\left(t_{k+1}^{N}\right)=M^{N}\left(t_{k+1}^{N}\right)$. We then show in the proof given in Section 5 that $L^{N}\left(t_{k}^{N}\right)>M^{N}\left(t_{k+1}^{N}\right)$ where $M^{N}\left(t_{k}^{N}\right) \geq F^{N}\left(t_{k}^{N}\right)$, so adoption must occur in all such periods, too.

Proposition 3.1. For any $\mathcal{T}^{N}$ there is a unique symmetric subgame perfect equilibrium $\sigma^{N}$. In that equilibrium, both firms $i, j \in\{1,2\}$ adopt immediately with probability 1 in any period $t_{k}^{N} \in \mathcal{T}^{N}$ with $M^{N}\left(t_{k}^{N}\right) \geq F^{N}\left(t_{k}^{N}\right)$, implying payoffs $V_{\sigma^{N}}^{i}\left(t_{k}^{N}\right)=M^{N}\left(t_{k}^{N}\right)$. No firm adopts if $M^{N}\left(t_{k}^{N}\right)<F^{N}\left(t_{k}^{N}\right)$ and $L^{N}\left(t_{k}^{N}\right) \leq V_{\sigma^{N}}^{i}\left(t_{k+1}^{N}\right)$, implying $V_{\sigma^{N}}^{i}\left(t_{k}^{N}\right)=V_{\sigma^{N}}^{i}\left(t_{k+1}^{N}\right)$. Otherwise, the firms adopt with probability

$$
\sigma_{j}^{N}(k)=\frac{L^{N}\left(t_{k}^{N}\right)-V_{\sigma^{N}}^{i}\left(t_{k+1}^{N}\right)}{L^{N}\left(t_{k}^{N}\right)-V_{\sigma^{N}}^{i}\left(t_{k+1}^{N}\right)+F^{N}\left(t_{k}^{N}\right)-M^{N}\left(t_{k}^{N}\right)}
$$

implying

$$
V_{\sigma^{N}}^{i}\left(t_{k}^{N}\right)=\frac{L^{N}\left(t_{k}^{N}\right) F^{N}\left(t_{k}^{N}\right)-V_{\sigma^{N}}^{i}\left(t_{k+1}^{N}\right) M^{N}\left(t_{k}^{N}\right)}{L^{N}\left(t_{k}^{N}\right)-V_{\sigma^{N}}^{i}\left(t_{k+1}^{N}\right)+F^{N}\left(t_{k}^{N}\right)-M^{N}\left(t_{k}^{N}\right)} \leq L^{N}\left(t_{k}^{N}\right) .
$$

The convergence analysis for the symmetric equilibria as $N \rightarrow \infty$ uses the following facts. As for the continuous-time model sketched in Figure 1, the equilibrium payoffs are at least $F^{N}$, 
which can be secured by waiting until $M^{N} \geq F^{N}$, as $F^{N}$ is nondecreasing before. Further, the indifference condition (3.2) implies that the increments of the equilibrium payoff process (3.4) are nonnegative where $F^{N}>M^{N}$ (see Lemma 5.2), where the payoffs are thus bounded by $M^{N}\left(T_{2}^{* N}\right)$. However, as the processes $L^{N}, F^{N}$ and $M^{N}$ approach their continuous-time limits (in a sense specified in Lemma 5.3), $V^{N}$ cannot stay in $(F, L)$ because its increments would sum up unboundedly. Thus the limit has to be $F$ where $F \leq L$. Where $F>L$, waiting will be dominant for any sufficiently fine grid. To simplify notation in the following convergence analysis, let $V_{\sigma^{N}}^{i}(t):=V_{\sigma^{N}}^{i}\left(t_{k}^{N}\right)$ for any $t \in \mathbb{R}_{+}$and $N \in \mathbb{N}$, where $t_{k}^{N} \leq t<t_{k+1}^{N}$.

Theorem 3.2. If $\sigma^{N}$ is the symmetric equilibrium on each $\mathcal{T}^{N}$, then $\lim _{N} V_{\sigma^{N}}^{i}(t)=F(t)$ for any $t \in \mathbb{R}_{+}$with $L(t) \geq F(t)$ and $\lim _{N} V_{\sigma^{N}}^{i}(t)=L\left(T_{1}\right)$ for any $t \in \mathbb{R}_{+}$with $L(t)<F(t)$. Further, for any fixed $t \in \mathbb{R}_{+}$and $l \in \mathbb{N}$ and $t_{k}^{N} \leq t<t_{k+1}^{N}$ for all $N \in \mathbb{N}$ we have

$$
\lim _{N \rightarrow \infty} \sigma_{i}^{N}(k+l)= \begin{cases}1 & \text { for } F(t)=M(t), \\ 0 & \text { for } F(t)>L(t), \\ \frac{L(t)-F(t)}{L(t)-M(t)} & \text { for } L(t) \geq F(t)>M(t) .\end{cases}
$$

\section{Equilibria with mixed strategies in continuous time}

As in FT, a mixed strategy for firm $i \in\{1,2\}$ for the game in continuous time is a family $G_{i}=\left(G_{i}^{t}\right)_{t \in \mathbb{R}_{+}}$of cumulative distribution functions $G_{i}^{t}$ on $\mathbb{R}_{+}$putting no weight on $[0, t)$, respectively, and that are time consistent in the sense of Bayes' law, i.e. that satisfy $G_{i}^{t}(u)=$ $G_{i}^{t}(v-)+\left(1-G_{i}^{t}(v-)\right) G_{i}^{v}(u)$ for all $t<v \leq u$, where $G_{i}^{t}(v-):=\lim _{s \nearrow v} G_{i}^{t}(s)$. Let $\Delta G_{i}^{t}(v):=$ $G_{i}^{t}(v)-G_{i}^{t}(v-)$ denote possible jumps due to atoms. Given any $i, j \in\{1,2\}, i \neq j$, and a mixed strategy profile $\left(G_{1}, G_{2}\right)$, the payoff to firm $i$ in the subgame beginning at $t \in \mathbb{R}_{+}$is

$$
\begin{aligned}
V\left(G_{i}^{t}, G_{j}^{t}\right) & =\int_{0}^{\infty}\left(1-G_{j}^{t}(u)\right) L(u) d G_{i}^{t}(u)+\int_{0}^{\infty}\left(1-G_{i}^{t}(u)\right) F(u) d G_{j}^{t}(u) \\
& +\sum_{u \in \mathbb{R}_{+}} \Delta G_{i}^{t}(u) \Delta G_{j}^{t}(u) M(u) .8
\end{aligned}
$$

The profile $\left(G_{1}, G_{2}\right)$ is a subgame perfect equilibrium if there is no $t \in \mathbb{R}_{+}$at which some firm $i$ could increase $V\left(G_{i}^{t}, G_{j}^{t}\right)$ by choosing any other distribution function than $G_{i}^{t}$ that puts no weight on $[0, t)$, i.e. if for all $t \in \mathbb{R}_{+},\left(G_{1}^{t}, G_{2}^{t}\right)$ is an equilibrium.

Consider Case B in Figure 1, i.e. $M\left(\hat{T}_{2}\right) \geq L\left(T_{1}^{*}\right)$, so that there are dates at which joint adoption gives a higher payoff than any leader payoff before. $S$ is the first time that joint adoption is at least as profitable as any earlier leader payoff. FT show that it is a subgame perfect equilibrium to fix any date in $\left[S, \hat{T}_{2}\right]$ and to adopt immediately from this date on, and

\footnotetext{
${ }^{8} V\left(G_{i}^{t}, G_{j}^{t}\right)$ differs only by the domain from $V\left(t_{i}, t_{j}\right)$ in Section 2 . This notation is consistent with FT.
} 
claim that the only alternative equilibria would be preemption.

The following Theorem 4.1 identifies additional equilibria using nondegenerate mixed strategies. In these equilibria, the firms also coordinate on a late joint adoption date $T$ after $S$. From some arbitrary earlier point $T_{0} \in\left[T_{1}, S\right]$ on, however, the firms start adopting at a rate that depends on (cumulative) increases in $L$. The associated risk to become follower makes the firms indifferent to adopt when $L$ is setting new records.

The principle behind Theorem 4.1 does not depend on the given payoff processes $L, F$ and $M$, in particular not on monotonicity. Therefore, the equilibrium verification step is formulated as a separate Proposition 5.5 in Section 5, with all requirements on $L, F$ and $M$ in its statement.

Theorem 4.1. Let $S:=\inf \left\{t \geq 0 \mid L(t)=M(t) \geq L\left(T_{1}^{*}\right)\right\}$ and $L^{*}(s):=\sup _{v \leq s} L(v)$. For any fixed $T_{0} \in\left[T_{1}, S\right]$ and $T \in\left[S, \hat{T}_{2}\right]$, the mixed strategies $\left(G_{1}, G_{2}\right)$ satisfying

$$
G_{1}^{t}(u)=G_{2}^{t}(u)=1-\mathbf{1}_{u<T} \exp \left(-\int_{t}^{u} \frac{\mathbf{1}_{s \geq T_{0}} d L^{*}(s)}{L(s)-F(s)}\right)
$$

for all $0 \leq t \leq u$ and $G_{1}^{t}(u)=G_{2}^{t}(u)=0$ for all $0 \leq u<t$ are a subgame perfect equilibrium, with payoff $V\left(G_{1}^{t}, G_{2}^{t}\right)=L\left(t \vee T_{0}\right)$ if $\max \left(t, T_{0}\right)<T_{1}^{*}$ and $V\left(G_{1}^{t}, G_{2}^{t}\right)=L(t \vee S)$ otherwise.

\section{Proofs}

The following lemma shows that the preemption equilibrium from FT cannot be sustained by strategies that are distribution functions over continuous time, see fn. 5 .

Lemma 5.1. For any $\varepsilon \in\left(0, T_{1}-T_{2}^{*}\right), \int_{T_{1}}^{T_{1}+\varepsilon}[L(t)-F(t)]^{-1} d L(t)=\infty$.

Proof. For any $t \in\left(T_{1}, T_{2}^{*}\right)$ we have $d F(t)=e^{-r t}\left(\pi_{0}(0)-\pi_{0}(1)\right) d t \geq 0$ and $L(t)>F(t)$, so

$$
\int_{T_{1}}^{T_{1}+\varepsilon} \frac{d L(t)}{L(t)-F(t)} \geq \int_{T_{1}}^{T_{1}+\varepsilon} \frac{d(L(t)-F(t))}{L(t)-F(t)}=\left.\ln (L(t)-F(t))\right|_{T_{1}} ^{T_{1}+\varepsilon}=\infty
$$

Note that similarly the result obtains more generally if there are $\lambda>-1$ and $\delta \in(0, \varepsilon]$ such that $F(t)-F\left(T_{1}\right) \geq-\lambda\left(L(t)-L\left(T_{1}\right)\right)$ for all $t \in\left(T_{1}, T_{1}+\delta\right)$ and $L\left(T_{1}+\delta\right)>L\left(T_{1}\right)$.

Proof of Proposition 3.1. We first argue that both firms must adopt immediately whenever $M^{N}\left(t_{k}^{N}\right)>F^{N}\left(t_{k}^{N}\right)$. Recall that $M^{N}$ is strictly decreasing on the set $\mathcal{T}^{N} \cap\left(\hat{T}_{2}^{N}, \infty\right)$, and that $L^{N}\left(t_{k}^{N}\right)-M^{N}\left(t_{k}^{N}\right) \rightarrow 0$ as $t_{k}^{N} \rightarrow \infty$. Thus, $L^{N}$ must have a maximum when restricted to that set and also one on the set $\mathcal{T}^{N} \cap\left(T_{2}^{* N}, \infty\right)$ that has finitely many elements more. Let $\hat{T}_{1}^{N}$ denote the latest time at which $L^{N}$ attains its maximum on the latter set. Then it holds at $t_{k}^{N}=\hat{T}_{1}^{N}$ that $L^{N}\left(t_{k}^{N}\right)>V_{\sigma^{N}}^{i}\left(t_{k+1}^{N}\right)$ for all strategy profiles $\sigma^{N}$ and firms $i \in\{1,2\}$ due to $L^{N}\left(t_{l}^{N}\right)>M^{N}\left(t_{l}^{N}\right) \geq F^{N}\left(t_{l}^{N}\right)$ for all $t_{l}^{N} \geq T_{2}^{* N}$. For any $t_{k}^{N}>T_{2}^{* N}$, indeed 
$M^{N}\left(t_{k}^{N}\right)>F^{N}\left(t_{k}^{N}\right)$ by strict quasiconcavity of (2.2). Thus, adopting is uniquely optimal at $t_{k}^{N}=\hat{T}_{1}^{N}$, implying $V_{\sigma^{N}}^{i}\left(\hat{T}_{1}^{N}\right)=M^{N}\left(\hat{T}_{1}^{N}\right)$ if $\sigma^{N}$ is a subgame perfect equilibrium. To work backwards, note that for any $t_{k}^{N} \geq T_{2}^{* N}$ we have

$$
\begin{aligned}
L^{N}\left(t_{k}^{N}\right)-M^{N}\left(t_{k+1}^{N}\right) & =\int_{t_{k}^{N}}^{t_{k+1}^{N}} e^{-r s}\left(\pi_{1}(1)-\pi_{0}(0)\right) d s-c\left(t_{k}^{N}\right)+c\left(t_{k+1}^{N}\right) \\
& >\int_{t_{k}^{N}}^{t_{k+1}^{N}} e^{-r s}\left(\pi_{1}(2)-\pi_{0}(1)\right) d s-c\left(t_{k}^{N}\right)+c\left(t_{k+1}^{N}\right) \\
& =M^{N}\left(t_{k}^{N}\right)-F^{N}\left(t_{k}^{N}\right) \geq 0 .
\end{aligned}
$$

Thus, if $T_{2}^{* N}<t_{k}^{N}<t_{k+1}^{N}=\hat{T}_{1}^{N}$ again $L^{N}\left(t_{k}^{N}\right)>V_{\sigma^{N}}^{i}\left(t_{k+1}^{N}\right)$, and by iteration immediate adoption must occur at any $t_{k}^{N} \in\left(T_{2}^{* N}, \hat{T}_{1}^{N}\right]$. The same argument applies to $\mathcal{T}^{N} \cap\left(\hat{T}_{1}^{N}, \infty\right)$ and so forth, to yield immediate adoption and $V_{\sigma^{N}}^{i}\left(t_{k}^{N}\right)=M^{N}\left(t_{k}^{N}\right)$ on all of $\mathcal{T}^{N} \cap\left(T_{2}^{* N}, \infty\right)$ in any subgame perfect equilibrium. Outside that set, $M^{N}\left(t_{k}^{N}\right)>F^{N}\left(t_{k}^{N}\right)$ can only occur at $t_{k}^{N}=T_{2}^{* N}$ by (3.1). Then again $L^{N}\left(t_{k}^{N}\right)>V_{\sigma^{N}}^{i}\left(t_{k+1}^{N}\right)$ by (5.1) and the firms must adopt immediately.

Next, if $M^{N}\left(t_{k}^{N}\right)=F^{N}\left(t_{k}^{N}\right)$, then $M^{N}\left(t_{k+1}^{N}\right)>F^{N}\left(t_{k+1}^{N}\right)$ by strict quasiconcavity of $(2.2)$ and thus $t_{k+1}^{N} \geq T_{2}^{* N}$ by (3.1), so at any such $t_{k}^{N}$ we still have $L^{N}\left(t_{k}^{N}\right)>V_{\sigma^{N}}^{i}\left(t_{k+1}^{N}\right)$ by (5.1). Then the LHS in (3.2) exceeds the RHS unless $\sigma_{j}^{N}(k)=1$, meaning that both firms must adopt in any symmetric equilibrium.

It remains to work backwards through the initial periods at which $M^{N}\left(t_{k}^{N}\right)<F^{N}\left(t_{k}^{N}\right)$, knowing $V_{\sigma^{N}}^{i}\left(t_{k+1}^{N}\right)=M^{N}\left(t_{k+1}^{N}\right)$ at the last of them. Considering (3.2), in equilibrium firm $i$ can only adopt in any of these periods if $L^{N}\left(t_{k}^{N}\right) \geq V_{\sigma^{N}}^{i}\left(t_{k+1}^{N}\right)$. In particular, if the latter binds, firm $i$ can only adopt if $\sigma_{j}^{N}(k)=0$, so also $\sigma_{i}^{N}(k)=0$ in a symmetric equilibrium. Thus, $V_{\sigma^{N}}^{i}\left(t_{k}^{N}\right)=V_{\sigma^{N}}^{i}\left(t_{k+1}^{N}\right)$ if $L^{N}\left(t_{k}^{N}\right) \leq V_{\sigma^{N}}^{i}\left(t_{k+1}^{N}\right)$. Finally, if $L^{N}\left(t_{k}^{N}\right)>V_{\sigma^{N}}^{i}\left(t_{k+1}^{N}\right)$, firm $i$ is indifferent only if (3.3) holds, the RHS of which now is in $(0,1)$. For any smaller (greater) $\sigma_{j}^{N}(k)$, firm $i$ must adopt (wait). Thus, in any symmetric equilibrium, $\sigma_{1}^{N}=\sigma_{2}^{N}$ must be given by (3.3), which plugged into (3.2) yields the payoff (3.4) in $\left(M^{N}\left(t_{k}^{N}\right), L^{N}\left(t_{k}^{N}\right)\right)$.

To prove Theorem 3.2, first three lemmas are established. A crucial fact for taking the limits of the symmetric equilibria as $N \rightarrow \infty$ is that the value processes $V_{\sigma^{N}}^{i}(\cdot)$ are nondecreasing and dominating $F^{N}(\cdot)$ up to $T_{2}^{* N}$. In particular we then have $F^{N}\left(t_{k}^{N}\right) \leq V_{\sigma^{N}}^{i}\left(t_{k}^{N}\right) \leq M^{N}\left(t_{l}^{N}\right)$ if $k \leq l$ and $t_{l}^{N}$ is the first period with $F^{N}\left(t_{l}^{N}\right) \leq M^{N}\left(t_{l}^{N}\right)$.

Lemma 5.2. If $\sigma^{N}$ is the symmetric equilibrium on $\mathcal{T}^{N}$, then $F^{N}\left(t_{k}^{N}\right) \leq V_{\sigma^{N}}^{i}\left(t_{k}^{N}\right) \leq V_{\sigma^{N}}^{i}\left(t_{k+1}^{N}\right)$ for all $t_{k}^{N} \in \mathcal{T}^{N}$ with $F^{N}\left(t_{k}^{N}\right)>M^{N}\left(t_{k}^{N}\right)$.

Proof. It suffices to show that $F^{N}\left(t_{k}^{N}\right) \leq V_{\sigma^{N}}^{i}\left(t_{k+1}^{N}\right)$ if $F^{N}\left(t_{k}^{N}\right)>M^{N}\left(t_{k}^{N}\right)$. Indeed, suppose the latter holds. If $L^{N}\left(t_{k}^{N}\right) \leq V_{\sigma^{N}}^{i}\left(t_{k+1}^{N}\right)$, then $\sigma_{1}^{N}(k)=\sigma_{2}^{N}(k)=0$ and $V_{\sigma^{N}}^{i}\left(t_{k}^{N}\right)=$ $V_{\sigma^{N}}^{i}\left(t_{k+1}^{N}\right) \geq F^{N}\left(t_{k}^{N}\right)$ as claimed. If $L^{N}\left(t_{k}^{N}\right)>V_{\sigma^{N}}^{i}\left(t_{k+1}^{N}\right)$, then $V_{\sigma^{N}}^{i}\left(t_{k}^{N}\right)$ is given by (3.4). 
The derivative of that term w.r.t. $V_{\sigma^{N}}^{i}\left(t_{k+1}^{N}\right)$ has the same sign as $F^{N}\left(t_{k}^{N}\right)-M^{N}\left(t_{k}^{N}\right)$ (recalling $\left.L^{N}\left(t_{k}^{N}\right)>M^{N}\left(t_{k}^{N}\right)\right)$, so $F^{N}\left(t_{k}^{N}\right)>M^{N}\left(t_{k}^{N}\right)$ and $V_{\sigma^{N}}^{i}\left(t_{k+1}^{N}\right) \geq F^{N}\left(t_{k}^{N}\right)$ imply $V_{\sigma^{N}}^{i}\left(t_{k}^{N}\right) \geq$ $F^{N}\left(t_{k}^{N}\right)$. (3.4) also implies

$$
V_{\sigma^{N}}^{i}\left(t_{k+1}^{N}\right)-V_{\sigma^{N}}^{i}\left(t_{k}^{N}\right)=\frac{\left(V_{\sigma^{N}}^{i}\left(t_{k+1}^{N}\right)-F^{N}\left(t_{k}^{N}\right)\right)\left(L^{N}\left(t_{k}^{N}\right)-V_{\sigma^{N}}^{i}\left(t_{k+1}^{N}\right)\right)}{L^{N}\left(t_{k}^{N}\right)-V_{\sigma^{N}}^{i}\left(t_{k+1}^{N}\right)+F^{N}\left(t_{k}^{N}\right)-M^{N}\left(t_{k}^{N}\right)},
$$

which shows that $V_{\sigma^{N}}^{i}\left(t_{k+1}^{N}\right) \geq V_{\sigma^{N}}^{i}\left(t_{k}^{N}\right)$ for $V_{\sigma^{N}}^{i}\left(t_{k+1}^{N}\right) \geq F^{N}\left(t_{k}^{N}\right)$ and $F^{N}\left(t_{k}^{N}\right)>M^{N}\left(t_{k}^{N}\right)$ also if $L^{N}\left(t_{k}^{N}\right)>V_{\sigma^{N}}^{i}\left(t_{k+1}^{N}\right)$.

To show now iteratively that $F^{N}\left(t_{k}^{N}\right) \leq V_{\sigma^{N}}^{i}\left(t_{k+1}^{N}\right)$ holds where $F^{N}\left(t_{k}^{N}\right)>M^{N}\left(t_{k}^{N}\right)$, consider the last such period, so $F^{N}\left(t_{k+1}^{N}\right) \leq M^{N}\left(t_{k+1}^{N}\right)=V_{\sigma^{N}}^{i}\left(t_{k+1}^{N}\right)$ and $t_{k+1}^{N} \leq T_{2}^{* N}$ by (3.1). If $t_{k+1}^{N}=T_{2}^{* N}$, then $F^{N}\left(t_{k}^{N}\right)-M^{N}\left(t_{k+1}^{N}\right)=\int_{t_{k}^{N}}^{t_{k+1}^{N}} e^{-r s}\left(\pi_{0}(1)-\pi_{0}(0)\right) d s \leq 0$, implying $F^{N}\left(t_{k}^{N}\right) \leq$ $V_{\sigma^{N}}^{i}\left(t_{k+1}^{N}\right)$. If $t_{k+1}^{N}<T_{2}^{* N}$, then $F^{N}\left(t_{k}^{N}\right)-F^{N}\left(t_{k+1}^{N}\right)=\int_{t_{k}^{N}}^{t_{k+1}^{N}} e^{-r s}\left(\pi_{0}(1)-\pi_{0}(0)\right) d s \leq 0$ and thus also $F^{N}\left(t_{k}^{N}\right) \leq V_{\sigma^{N}}^{i}\left(t_{k+1}^{N}\right)$. In either case then $F^{N}\left(t_{k}^{N}\right) \leq V_{\sigma^{N}}^{i}\left(t_{k}^{N}\right)$ as shown before. Moving further backward, then $t_{k+1}^{N}<T_{2}^{* N}$ must hold and hence $F^{N}\left(t_{k}^{N}\right) \leq F^{N}\left(t_{k+1}^{N}\right) \leq V_{\sigma^{N}}^{i}\left(t_{k+1}^{N}\right)$ as just established. (As a side product this proves also that $F^{N}\left(t_{k}^{N}\right) \leq F^{N}\left(t_{k+1}^{N}\right)$ whenever $F^{N}\left(t_{k+1}^{N}\right) \geq M^{N}\left(t_{k+1}^{N}\right)$.)

The discrete-time payoff processes converge as follows to their continuous-time counterparts. $M^{N}$ converges uniformly to $M$ as $N \rightarrow \infty$ in the sense that $\sup \left\{\left|M^{N}\left(t_{k}^{N}\right)-M\left(t_{k}^{N}\right)\right| ; k \in\right.$ $\left.\mathbb{N}_{0}\right\} \rightarrow 0$, because this sequence is identically $0 . L^{N}$ converges in the same notion, though not trivially. $F^{N}$ converges pointwise if one considers the grid points close to a fixed time $t \in \mathbb{R}_{+}$, and so do $L^{N}$ and $M^{N}$, too.

Lemma 5.3. $\sup \left\{\left|L^{N}\left(t_{k}^{N}\right)-L\left(t_{k}^{N}\right)\right| ; k \in \mathbb{N}_{0}\right\} \rightarrow 0$ as $N \rightarrow \infty$. Further, for any fixed $t \in \mathbb{R}_{+}$ and $l \in \mathbb{N}_{0}$, if $t_{k}^{N} \leq t<t_{k+1}^{N}$ for all $N \in \mathbb{N}$, then $F^{N}\left(t_{k+l}^{N}\right) \rightarrow F(t), L^{N}\left(t_{k+l}^{N}\right) \rightarrow L(t)$ and $M^{N}\left(t_{k+l}^{N}\right) \rightarrow M(t)$ as $N \rightarrow \infty$.

Proof. The first claim follows from $\sup \left\{\left|L^{N}\left(t_{k}^{N}\right)-L\left(t_{k}^{N}\right)\right| ; k \in \mathbb{N}_{0}\right\} \leq\left(\pi_{1}(1)-\pi_{1}(2)\right)\left\|\mathcal{T}^{N}\right\|$ for all $N \in \mathbb{N}$, which holds because $L^{N}\left(t_{k}^{N}\right)$ and $L\left(t_{k}^{N}\right)$ differ only by the revenue $\pi_{1}(1)-\pi_{1}(2)$ between disagreeing follower reaction times, which is for at most one period.

For $F^{N}$ also the cost $c$ matters. To obtain pointwise convergence, fix some $t \in \mathbb{R}_{+}$and choose for each grid $\mathcal{T}^{N}$ the periods satisfying $t_{k}^{N} \leq t<t_{k+1}^{N}$. Then

$$
\begin{aligned}
F^{N}\left(t_{k}^{N}\right)-F(t) & =\int_{t_{k}^{N}}^{t} e^{-r s}\left(\pi_{0}(1)-\pi_{0}(0)\right) d s+\int_{T_{2}^{*} \vee t}^{T_{2}^{* N} \vee t_{k+1}^{N}} e^{-r s}\left(\pi_{0}(1)-\pi_{1}(2)\right) d s \\
& -c\left(T_{2}^{* N} \vee t_{k+1}^{N}\right)+c\left(T_{2}^{*} \vee t\right) .
\end{aligned}
$$

The adoption times differ again by at most one period, $\left|\left(T_{2}^{* N} \vee t_{k+1}^{N}\right)-\left(T_{2}^{*} \vee t\right)\right| \leq\left\|\mathcal{T}^{N}\right\|$, because if $T_{2}^{*}<t_{k+1}^{N}$, then $t_{k+1}^{N} \geq T_{2}^{* N}$, and if $T_{2}^{*} \geq t_{k+1}^{N}$, then $t_{k+1}^{N} \leq T_{2}^{* N}$ and $\mid T_{2}^{* N}-$ 
$T_{2}^{*} \mid \leq\left\|\mathcal{T}^{N}\right\|$. Thus $F^{N}\left(t_{k}^{N}\right) \rightarrow F(t)$ as $N \rightarrow \infty$ by continuity of $c$. Analogously, also $F^{N}\left(t_{k+l}^{N}\right) \rightarrow F(t), L^{N}\left(t_{k+l}^{N}\right) \rightarrow L(t)$ and $M^{N}\left(t_{k+l}^{N}\right) \rightarrow M(t)$ for the same $\left(t_{k}^{N}\right)_{N}$ and any fixed $l \in \mathbb{N}_{0}$, as then $\left|\left(T_{2}^{* N} \vee t_{k+l+1}^{N}\right)-\left(T_{2}^{*} \vee t\right)\right| \leq(l+1)\left\|\mathcal{T}^{N}\right\|$.

An immediate consequence of Lemmas 5.2 and 5.3 is that $\liminf _{N} V_{\sigma^{N}}^{i}(t) \geq F(t)$ for any $t \in \mathbb{R}_{+}$. The following lemma shows that due to the increments of $V_{\sigma^{N}}^{i}(\cdot)$ implied by (3.4), any cluster point above $F(t)$ must imply an immediate jump above $L(t)$ and thus the lim sup would have to stay above $L$. The latter will subsequently be shown to be impossible given the boundedness of $V_{\sigma^{N}}^{i}(\cdot)$ observed in the context of Lemma 5.2.

Lemma 5.4. If $\sigma^{N}$ is the symmetric equilibrium on each $\mathcal{T}^{N}$ and $\lim \sup _{N} V_{\sigma^{N}}^{i}(t)>F(t)$ for some $t \in \mathbb{R}_{+}$with $L(t)>F(t)$, then $\lim \sup _{N} V_{\sigma^{N}}^{i}(s) \geq L(s)$ for all $s>t$ with $L(s)>F(s)$.

Proof. Suppose that for some $t<T_{2}^{*},\left(V_{\sigma^{N}}^{i}(t)\right)_{N}$ has a cluster point $C \in(F(t), L(t))$. We first show that any subsequence of $\left(V_{\sigma^{N}}^{i}(\cdot)\right)_{N}$ that converges to the cluster point $C$ at $t$ must have a lim inf immediately to the right of $t$ not less than $L(t)$, as otherwise there would be some interval after $t$ in which the increments of $V_{\sigma^{N}}^{i}(\cdot)$ given by (5.2) are bounded away from 0 as they become arbitrarily frequent. Therefore, choose arbitrary $0<\varepsilon<\min (L(t)-C, C-F(t))$ and $0<\delta<\min \left(\varepsilon, T_{2}^{*}-t\right)$ such that $|F(s)-F(t)|<\varepsilon$ and $|L(s)-L(t)|<\varepsilon$ for all $|s-t| \leq \delta$ by continuity. By monotonicity of $V_{\sigma^{N}}^{i}(\cdot)$, any subsequence converging to the cluster point $C$ at $t$ must have a liminf not below $C$ at $t+\delta$. Suppose by way of contradiction that one has a cluster point $D<L(t)-\varepsilon$. For any subsequence converging both to $C$ at $t$ and $D$ at $t+\delta, V_{\sigma^{N}}^{i}(t)$ is arbitrarily close to $C$ and $V_{\sigma^{N}}^{i}(t+\delta)$ arbitrarily close to $D$ for all $N$ sufficiently large, and so $\max \left(V_{\sigma^{N}}^{i}(s)-D, C-V_{\sigma^{N}}^{i}(s), 0\right)$ is arbitrarily close to 0 for all $s \in[t, t+\delta]$ by monotonicity of $V_{\sigma^{N}}^{i}(\cdot)$. By uniform convergence, $L^{N}\left(t_{k}^{N}\right)$ is arbitrarily close to $L\left(t_{k}^{N}\right)>L(t)-\varepsilon$ for all $t_{k}^{N} \in[t, t+\delta]$ for all $N$ sufficiently large. Recall that $F^{N}\left(t_{k}^{N}\right) \leq F\left(t_{k}^{N}\right)<F(t)+\varepsilon<C$ for all $t_{k}^{N} \in[t, t+\delta]$, so in particular $F^{N}\left(t_{k}^{N}\right)-M^{N}\left(t_{k}^{N}\right) \leq F\left(t_{k}^{N}\right)-M\left(t_{k}^{N}\right)$ is bounded for all $t_{k}^{N} \in[t, t+\delta]$ by continuity of $F(\cdot)-M(\cdot)$, but nonnegative due to $t_{k}^{N}<T_{2}^{*}$. Together, if $D<L(t)-\varepsilon$, then the increments of $V_{\sigma^{N}}^{i}\left(t_{k}^{N}\right)$ given by (5.2) are positive and bounded away from 0 for all $t_{k}^{N}, t_{k+1}^{N} \in[t, t+\delta]$ for all $N$ sufficiently large, and there are arbitrarily many of them in that interval as $\left\|\mathcal{T}^{N}\right\| \rightarrow 0$, which contradicts that $V_{\sigma^{N}}^{i}(t+\delta)$ has the cluster point $D$. As $\varepsilon$ was arbitrary, the liminf at any $s>t$ of any subsequence of $V_{\sigma^{N}}^{i}(\cdot)$ that converges to the cluster point $C$ at $t$ cannot be below $L(t)$.

By continuity of $L(\cdot)$ and $F(\cdot)$ and monotonicity of all $V_{\sigma^{N}}^{i}(\cdot)$ this implies that if ever $\lim \sup _{N} V_{\sigma^{N}}^{i}(t)>F(t)$ for some $t$ with $L(t)>F(t)$, then $\lim \sup _{N} V_{\sigma^{N}}^{i}(s) \geq L(s)$ for all $s>t$ with $L(s)>F(s)$.

Proof of Theorem 3.2. By Lemma 5.4, if $\lim _{\sup _{N}} V_{\sigma^{N}}^{i}(t)>F(t)$ for some $t$ with $L(t)>$ $F(t)$, i.e. $t \in\left(T_{1}, T_{2}^{*}\right)$, then $\lim \sup _{N} V_{\sigma^{N}}^{i}(s)>M\left(T_{2}^{*}\right)$ for some $s \in\left[t, T_{2}^{*}\right)$, because $L(s)>$ $M\left(T_{2}^{*}\right) \geq F(s)$ for all $s$ in $\left[T_{2}^{*}-\varepsilon, T_{2}^{*}\right)$ with sufficiently small $\varepsilon>0$. However, if $s \leq T_{2}^{*}$ then 
$\lim \sup _{N} V_{\sigma^{N}}^{i}(s) \leq M\left(T_{2}^{*}\right)$, because $V_{\sigma^{N}}^{i}(\cdot)$ is nondecreasing, and for $t_{k}^{N} \leq s=T_{2}^{*}<t_{k+1}^{N}$ one of the two grid points is the first time that $F^{N}\left(t_{l}^{N}\right) \leq M^{N}\left(t_{l}^{N}\right)$ (cf. the remark before Lemma $5.2)$, such that then $V_{\sigma^{N}}^{i}\left(t_{k}^{N}\right) \leq \max \left(M^{N}\left(t_{k}^{N}\right), M^{N}\left(t_{k+1}^{N}\right)\right)$, which converges to $M\left(T_{2}^{*}\right)$.

Therefore we must have $\lim \sup _{N} V_{\sigma^{N}}^{i}(t)=F(t)$ for all $t \in \mathbb{R}_{+}$with $L(t)>F(t)$ and thus convergence by $\lim \inf _{N} V_{\sigma^{N}}^{i}(t) \geq F(t)$. The same argument shows convergence for $t=T_{1}$, because if $\lim \sup _{N} V_{\sigma^{N}}^{i}(t)>F(t)$ for $t=T_{1}$, then this would also hold for some $t>T_{1}$ with $L(t)>F(t)$ by continuity of $F(\cdot)$.

By continuity of the limit in $t$ and monotonicity of $V_{\sigma^{N}}^{i}(\cdot), \lim _{N} V_{\sigma^{N}}^{i}\left(t_{k}^{N}\right)=\lim _{N} V_{\sigma^{N}}^{i}\left(t_{k+l}^{N}\right)$ for any $t \in \mathbb{R}_{+}$and $t_{k}^{N} \leq t<t_{k+1}^{N}$ and fixed $l \in \mathbb{N}$. Therefore, and by Lemma 5.3,

$$
\lim _{N \rightarrow \infty} \sigma_{i}^{N}(k)=\lim _{N \rightarrow \infty} \sigma_{i}^{N}(k+l)=\frac{L(t)-F(t)}{L(t)-M(t)}
$$

where $L(t)>F(t)$. At $t=T_{1}$, we have $L\left(T_{1}\right)=\lim _{N} L^{N}\left(t_{k+l}^{N}\right)>M\left(T_{1}\right)=\lim _{N} M^{N}\left(t_{k+l}^{N}\right)$ for $t_{k}^{N} \leq T_{1}<t_{k+1}^{N}$. Thus, considering (3.2), if $\lim _{\sup _{N}} \sigma_{i}^{N}(k+l)>0$, this would mean $\lim \inf _{N} V_{\sigma^{N}}^{i}\left(t_{k+l}^{N}\right)<L\left(T_{1}\right)=F\left(T_{1}\right)$, which is not true. Therefore $\lim _{N} \sigma_{i}^{N}(k+l)=0$.

Besides $t=T_{1}$, we have $L(t)=F(t)$ for $t \geq T_{2}^{*}$, i.e. $F(t)=M(t)$. By $\lim \sup _{N} V_{\sigma^{N}}^{i}\left(T_{2}^{*}\right) \leq$ $M\left(T_{2}^{*}\right)$ as argued above and $\liminf _{N} V_{\sigma^{N}}^{i}\left(T_{2}^{*}\right) \geq F\left(T_{2}^{*}\right)=M\left(T_{2}^{*}\right)$, we also have convergence for $t=T_{2}^{*}$. With $t=T_{2}^{*}<t_{k+1}^{N}$, we have $T_{2}^{* N} \leq t_{k+1}^{N}$ and thus $\sigma_{i}^{N}(k+l)=1$ for any $l \in \mathbb{N}$. If $T_{2}^{*}<t<t_{k+1}^{N}$, then $t_{k}^{N} \geq T_{2}^{* N}$ for all $N$ sufficiently large and thus $\sigma^{N}(k)=\sigma^{N}(k+l)=1$ for all $l \in \mathbb{N}$ and $V_{\sigma^{N}}^{i}\left(t_{k}^{N}\right)=M^{N}\left(t_{k}^{N}\right)$, converging to $M(t)=F(t)$.

Now consider an arbitrary $t<T_{1}$, i.e. $L(t)<F(t)$. With $t_{k}^{N} \leq t<t_{k+1}^{N}$, we have $M^{N}\left(t_{k}^{N}\right)<L^{N}\left(t_{k}^{N}\right)<F^{N}\left(t_{k}^{N}\right) \leq V_{\sigma^{N}}^{i}\left(t_{k+1}^{N}\right)$ for all $N$ sufficiently large by Lemmas 5.2 and 5.3 and thus $\sigma_{i}^{N}(k)=0$ and $V_{\sigma^{N}}^{i}\left(t_{k}^{N}\right)=V_{\sigma^{N}}^{i}\left(t_{k+1}^{N}\right)$. With $t_{k}^{N}<T_{1}<T_{1}^{*}$, further $L^{N}\left(t_{m}^{N}\right)<L^{N}\left(t_{k}^{N}\right)$ and thus $\sigma_{i}^{N}(m)=0$ and $V_{\sigma^{N}}^{i}\left(t_{m}^{N}\right)=V_{\sigma^{N}}^{i}\left(t_{k+1}^{N}\right)$ for all $m<k$. That means $\lim \inf _{N} V_{\sigma^{N}}^{i}(s) \geq F(t)$ for all $s \leq t$. As $t<T_{1}$ was arbitrary and $F(t)$ increases continuously to $F\left(T_{1}\right), \liminf _{N} V_{\sigma^{N}}^{i}(t) \geq F\left(T_{1}\right)$ for all $t<T_{1}$. However, by monotonicity of $V_{\sigma^{N}}^{i}(\cdot), \lim \sup _{N} V_{\sigma^{N}}^{i}(t) \leq \lim _{N} V_{\sigma^{N}}^{i}\left(T_{1}\right)=F\left(T_{1}\right)=L\left(T_{1}\right)$, so convergence holds. Finally fix any $s<t<T^{1}$ and $l \in \mathbb{N}$. Then $\lim _{N} \sigma_{i}^{N}(k+l)=0$ for $t_{k}^{N} \leq s<t_{k+1}^{N}$, as $t_{k+l}^{N}<t$ for sufficiently large $N$, which implies $\sigma_{i}^{N}(k+l)=0$ as argued before.

The verification of the equilibria from Theorem 4.1 does not depend on the particular properties of $L, F$ and $M$ from Section 2. Therefore, these may be any functions of time in the following Proposition 5.5, which is applied in the subsequent proof of Theorem 4.1. Recall the notation $G_{i}^{t}(u-):=\lim _{s \nearrow u} G^{t}(s)$ and $\Delta G_{i}^{t}(u)=G_{i}^{t}(u)-G_{i}^{t}(u-)$ that will be used for any monotone function.

Proposition 5.5. Consider any times $0 \leq t \leq T_{0} \leq T \leq \inf \{s \geq t \mid L(s)<F(s)\}$. Suppose that $L^{*}(s):=\sup _{v \leq s} L(v)$ is continuous in $s \in\left[T_{0}, T\right)$ and that $\left(G_{1}^{T}, G_{2}^{T}\right)$ is an equilibrium for the subgame beginning at $T \in \mathbb{R}_{+}$with symmetric payoffs $V\left(G_{1}^{T}, G_{2}^{T}\right)=V\left(G_{2}^{T}, G_{1}^{T}\right)$. 
Then the mixed strategies $G_{1}^{t}$ and $G_{2}^{t}$ satisfying

$$
G_{i}^{t}(u)=1-\exp \left(-\int_{t}^{u} \frac{\mathbf{1}_{s \geq T_{0}} d L^{*}(s)}{L(s)-F(s)}\right)
$$

for all $u \in[0, T)$ and $G_{i}^{t}(u)=G_{i}^{t}(T-)+\left(1-G_{i}^{t}(T-)\right) G_{i}^{T}(u)$ for all $u \geq T, i=1,2$, are an equilibrium in the subgame beginning at $t$ if and only if $\left(1-G_{1}^{t}(T-)\right)\left(V\left(G_{1}^{T}, G_{2}^{T}\right)-\right.$ $\left.\sup _{t \leq v<T} L(v)\right) \geq 0$ and $\Delta G_{1}^{t}(M-F) \geq 0$ on $[t, T)$, both with equality if $G_{1}^{t}$ increases continuously somewhere before $T$.

The corresponding symmetric payoffs are $V\left(G_{1}^{t}, G_{2}^{t}\right)=L^{*}\left(T_{0}\right)$ if $G_{1}^{t}$ increases continuously somewhere before $T$ and otherwise $V\left(G_{1}^{t}, G_{2}^{t}\right)=V\left(G_{1}^{T}, G_{2}^{T}\right)$ if $G_{1}^{t}(T-)=0$ and $V\left(G_{1}^{t}, G_{2}^{t}\right)=$ $M\left(\inf \left\{u \geq t \mid G_{1}^{t}(u)=1\right\}\right)$ if $G_{1}^{t}(T-)=1$.

The strategies $G_{i}^{t}$ can only have a jump before $T$ if $d L^{*}>0$ and $L=F$, which will thus be terminal, and where joint adoption must be an equilibrium. If $\int_{\left[T_{0}, T\right)}[L(s)-F(s)]^{-1} d L^{*}(s)$ is finite, then the continuation equilibrium payoff $V\left(G_{1}^{T}, G_{2}^{T}\right)$ must be sufficiently high, whereas if the integral is unbounded but continuous, then $V\left(G_{1}^{T}, G_{2}^{T}\right)$ does not matter.

Proof of Proposition 5.5. Each $G_{i}^{t}$ is right-continuous and nondecreasing as $L^{*}(s)$ is continuous and nondecreasing and $L(s) \geq F(s)$ for all $s \in\left[T_{0}, T\right)$. Also $G_{i}^{t}(u)=0$ for $u<t$ and $\lim _{u \rightarrow \infty} G_{i}^{t}(u)=\lim _{u \rightarrow \infty} G_{i}^{T}(u)=1 . G_{i}^{t}$ is a best reply to $G_{j}^{t}$ for any $i, j \in\{1,2\}, i \neq j$, if and only if $V\left(u, G_{j}^{t}\right):=V\left(\mathbf{1}_{s \geq u}, G_{j}^{t}\right) \leq V\left(G_{i}^{t}, G_{j}^{t}\right)$ for all $u \geq t$, and if equality holds for all $u$ in the support of $d G_{i}^{t}$. Note that

$$
V\left(u, G_{j}^{t}\right)=\int_{[0, u)} F(s) d G_{j}^{t}(s)+\Delta G_{j}^{t}(u) M(u)+\left(1-G_{j}^{t}(u)\right) L(u) .
$$

Letting $\tau_{j}^{G}:=\inf \left\{u \in \mathbb{R}_{+} \mid G_{j}^{t}(u)=1\right\}, V\left(u, G_{j}^{t}\right)$ is constant for $u>\tau_{j}^{G}$.

First consider $G_{j}^{t}(T-)=1$, so in fact $G_{1}^{t}=G_{2}^{t}$. Suppose $\Delta G_{j}^{t}\left(\tau_{j}^{G}\right)=1\left(\right.$ so $\left.\tau_{j}^{G}<T\right)$. Then $L\left(\tau_{j}^{G}\right)=F\left(\tau_{j}^{G}\right)$ and $d A\left(\tau_{j}^{G}\right)>0$, so $A\left(\tau_{j}^{G}\right)=L^{*}\left(\tau_{j}^{G}\right)-L^{*}\left(T_{0}\right)=L\left(\tau_{j}^{G}\right)-L^{*}\left(T_{0}\right)$ and thus $L\left(\tau_{j}^{G}\right)=L^{*}\left(\tau_{j}^{G}\right)$. By $V\left(G_{i}^{t}, G_{j}^{t}\right)=M\left(\tau_{j}^{G}\right)$ and $V\left(u, G_{j}^{t}\right)=F\left(\tau_{j}^{G}\right)$ for all $u>\tau_{j}^{G}$, we need $M\left(\tau_{j}^{G}\right) \geq F\left(\tau_{j}^{G}\right)$. This then ensures also $V\left(G_{i}^{t}, G_{j}^{t}\right) \geq V\left(u, G_{j}^{t}\right)=L(u)$ for all $u<\tau_{j}^{G}$ by $F\left(\tau_{j}^{G}\right)=L\left(\tau_{j}^{G}\right)=L^{*}\left(\tau_{j}^{G}\right)$.

Now suppose $\Delta G_{j}^{t}\left(\tau_{j}^{G}\right)<1$, i.e. $G_{j}^{t}$ increases continuously before $\tau_{j}^{G} \leq T$. Letting $A(u):=$ $\int_{t}^{u} \mathbf{1}_{s \geq T_{0}} d L^{*}(s)=L^{*}\left(u \vee T_{0}\right)-L^{*}\left(T_{0}\right), G_{j}^{t}$ given by (5.4) satisfies $(L(s)-F(s)) d G_{j}^{t}(s)=$ $\left(1-G_{j}^{t}(s)\right) d A(s)$ for any $s \leq u<\min \left(\tau_{j}^{G}, T\right)$. Applying integration by parts to the RHS and using continuity of $G_{j}^{t}(s)$ and $A(s)$ and $G_{j}^{t}(0)=A(0)=0$ yields

$$
\int_{[0, u)} F(s) d G_{j}^{t}(s)=\int_{[0, u)}(L(s)-A(s)) d G_{j}^{t}(s)-\left(1-G_{j}^{t}(u)\right) A(u) .
$$

Here $d G_{j}^{t}(s)>0$ only if $d A(s)>0$, implying again $A(s)=L^{*}(s)-L^{*}\left(T_{0}\right)=L(s)-L^{*}\left(T_{0}\right)$. 
For $u<\min \left(\tau_{j}^{G}, T\right)$ also $\Delta G_{j}^{t}(u)=0$ and thus $V\left(u, G_{j}^{t}\right)=L^{*}\left(T_{0}\right) G_{j}^{t}(u)+\left(1-G_{j}^{t}(u)\right)(L(u)-$ $A(u)) \leq L^{*}\left(T_{0}\right)$, with equality if $d A(u)>0$, i.e. if $d G_{i}^{t}(u)>0$. If further $\Delta G_{j}^{t}\left(\tau_{j}^{G}\right)=0$, then indeed $V\left(G_{i}^{t}, G_{j}^{t}\right)=L^{*}\left(T_{0}\right)=V\left(u, G_{j}^{t}\right)$ for all $u \geq \tau_{j}^{G}$.

If $0<\Delta G_{j}^{t}\left(\tau_{j}^{G}\right)<1$, then $u=\tau_{j}^{G}<T$ is in the support of $d G_{i}^{t}$, but no $u>\tau_{j}^{G}$. By the previous arguments $V\left(\tau_{j}^{G}, G_{j}^{t}\right)=L^{*}\left(T_{0}\right) G_{j}^{t}\left(\tau_{j}^{G}-\right)+\Delta G_{j}^{t}\left(\tau_{j}^{G}\right)\left(M\left(\tau_{j}^{G}\right)-A\left(\tau_{j}^{G}\right)\right)$ and, for any $u>\tau_{j}^{G}, V\left(u, G_{j}^{t}\right)=L^{*}\left(T_{0}\right) G_{j}^{t}\left(\tau_{j}^{G}-\right)+\Delta G_{j}^{t}\left(\tau_{j}^{G}\right)\left(F\left(\tau_{j}^{G}\right)-A\left(\tau_{1}^{G}\right)\right)$. Thus $V\left(\tau_{j}^{G}, G_{j}^{t}\right) \geq V\left(u, G_{j}^{t}\right)$ if and only if $M\left(\tau_{j}^{G}\right) \geq F\left(\tau_{j}^{G}\right)$. Then, noting that we can have $\Delta G_{j}^{t}\left(\tau_{j}^{G}\right)>0$ and $\tau_{j}^{G}<T$ only if $d A\left(\tau_{j}^{G}\right)>0$, whence again $A\left(\tau_{j}^{G}\right)=L\left(\tau_{j}^{G}\right)-L^{*}\left(T_{0}\right)$, and only if $L\left(\tau_{j}^{G}\right)=F\left(\tau_{j}^{G}\right)$, we have $V\left(\tau_{j}^{G}, G_{j}^{t}\right)=L^{*}\left(T_{0}\right) G_{j}^{t}\left(\tau_{j}^{G}-\right)+\Delta G_{j}^{t}\left(\tau_{j}^{G}\right)\left(M\left(\tau_{j}^{G}\right)-F\left(\tau_{j}^{G}\right)+L^{*}\left(T_{0}\right)\right) \geq L^{*}\left(T_{0}\right)$. In fact, equality must hold by $L^{*}\left(T_{0}\right)=V\left(u, G_{j}^{t}\right)$ for all $u<\tau_{j}^{G}$ in the support of $d G_{i}^{t}$ as shown above, i.e. $M\left(\tau_{1}^{G}\right)=F\left(\tau_{1}^{G}\right)$.

Now consider $G_{j}^{t}(T-)<1$. Then $G_{1}^{t}(T-)=G_{2}^{t}(T-)<1$ and some $u \geq T$ is in the support of $d G_{i}^{t}$. As $\left(G_{1}^{T}, G_{2}^{T}\right)$ is an equilibrium, $V\left(u, G_{j}^{t}\right) \leq V\left(G_{i}^{T}, G_{j}^{t}\right)$ for all $u \geq T$, with equality for $u$ in the support of $d G_{i}^{t}$. If $G_{j}^{t}(T-)=0$, then $G_{1}^{t}=G_{1}^{T}$ and $G_{2}^{t}=G_{2}^{T}$ and $V\left(G_{i}^{t}, G_{j}^{t}\right)=$ $V\left(G_{i}^{T}, G_{j}^{T}\right) \geq V\left(u, G_{j}^{t}\right)$ also for all $u \in[t, T)$ if and only if $V\left(G_{i}^{T}, G_{j}^{T}\right) \geq \sup _{t \leq u<T} L(u)$. If $0<G_{j}^{t}(T-)<1$, then we have $V\left(u, G_{j}^{t}\right)=L^{*}\left(T_{0}\right)$ for some $u<T$ in the support of $d G_{i}^{t}$ as shown above. Thus we need $V\left(G_{i}^{T}, G_{j}^{t}\right)=L^{*}\left(T_{0}\right)$. Now $V\left(G_{i}^{T}, G_{j}^{t}\right)=\int_{[0, T)} F(s) d G_{j}^{t}(s)+(1-$ $\left.G_{j}^{t}(T-)\right) V\left(G_{i}^{T}, G_{j}^{T}\right)=L^{*}\left(T_{0}\right) G_{j}^{t}(T-)+\left(1-G_{j}^{t}(T-)\right)\left(V\left(G_{i}^{T}, G_{j}^{T}\right)-A(T-)\right)$ by the arguments from above. Therefore $V\left(G_{i}^{T}, G_{j}^{t}\right)=L^{*}\left(T_{0}\right)$ if and only if $V\left(G_{1}^{T}, G_{2}^{T}\right)=L^{*}(T-)$, which now exceeds $L^{*}\left(T_{0}\right)$ and thus $L^{*}(T-)=\sup _{t \leq u<T} L(u)$.

Proof of Theorem 4.1. Any $G_{1}^{t}$ is right-continuous and nondecreasing as $L^{*}(s)$ is continuous and nondecreasing and $L(s) \geq F(s)$ for all $s \geq T_{0} \geq T_{1}$. Further $\lim _{u \rightarrow \infty} G_{1}^{t}(u)=1$ as $T \leq \hat{T}_{2}<\infty$. The family $G_{1}$ is also time consistent, because the time consistency condition can be written as $\left(1-G_{1}^{t}(u)\right)=\left(1-G_{1}^{t}(v-)\right)\left(1-G_{1}^{v}(u)\right)$ for any $t \leq v \leq u$ and holds by

$$
\begin{aligned}
& \mathbf{1}_{u<T} \exp \left(-\int_{t}^{u} \frac{\mathbf{1}_{s \geq T_{0}} d L^{*}(s)}{L(s)-F(s)}\right) \\
& =\mathbf{1}_{v \leq T} \exp \left(-\int_{t}^{v} \frac{\mathbf{1}_{s \geq T_{0}} d L^{*}(s)}{L(s)-F(s)}\right) \mathbf{1}_{u<T} \exp \left(-\int_{v}^{u} \frac{\mathbf{1}_{s \geq T_{0}} d L^{*}(s)}{L(s)-F(s)}\right) \\
& =\mathbf{1}_{u<T} \exp \left(-\int_{t}^{v} \frac{\mathbf{1}_{s \geq T_{0}} d L^{*}(s)}{L(s)-F(s)}\right) \exp \left(-\int_{v}^{u} \frac{\mathbf{1}_{s \geq T_{0}} d L^{*}(s)}{L(s)-F(s)}\right) .
\end{aligned}
$$

For any $t \geq S \geq \max \left(T_{0}, T_{2}^{*}\right)$, we have $G_{1}^{t}(t)=G_{2}^{t}(t)=1$, either due to $t \geq T$ or due to $d L^{*}(t)=d M(t)>0$ and $L(t)=F(t)$. These are an equilibrium by $M(t) \geq F(t)$.

To show that $\left(G_{1}^{t}, G_{2}^{t}\right)$ are also an equilibrium for any $t<S$, we can now apply Proposition 5.5 with $S$ in place of $T$ and $\max \left(t, T_{0}\right)$ in place of $T_{0}$, and using the continuation equilibrium with symmetric payoffs $V\left(G_{1}^{S}, G_{2}^{S}\right)=V\left(G_{2}^{S}, G_{1}^{S}\right)=M(S)$. Then all hypotheses are satisfied. Indeed, $G_{1}^{t}(u)=G_{2}^{t}(u)$ are continuous before $S$, which is the first time with $d L^{*}>0$ and $L=F$, and where indeed $V\left(G_{1}^{S}, G_{2}^{S}\right)=M(S)=L^{*}(S) \geq L(v)$ for all $v \in[t, S)$ by definition 
of $S$.

The payoffs for $t<S$ are as claimed by Proposition 5.5. If $\max \left(t, T_{0}\right) \geq T_{1}^{*}$, we have $G_{1}^{t}(S-)=0$ and the equilibrium payoff at $S$ is $M(S)=L(S)$. If $\max \left(t, T_{0}\right)<T_{1}^{*}, G_{1}^{t}$ increases continuously before $S$ as $L$ does on $\left[0, T_{1}^{*}\right]$, which implies also $L^{*}\left(t \vee T_{0}\right)=L\left(t \vee T_{0}\right)$.

\section{References}

Azevedo, A. and D. Paxson (2014). Developing real option game models. Eur. J. Oper. Res. 23\%, 909-920.

Fudenberg, D. and D. Levine (1986). Limit games and limit equilibria. J. Econ. Theory 38, 261-279.

Fudenberg, D. and D. Levine (2009). Repeated games with frequent signals. Q. J. Econ. 124, 233-265.

Fudenberg, D. and J. Tirole (1985). Preemption and rent equalization in the adoption of new technology. Rev. Econ. Stud. 52(3), 383-401.

Grenadier, S. R. (1996). The strategic exercise of options: Development cascades and overbuilding in real estate markets. J. Finance 51(5), 1653-1679.

Hendricks, K. and C. Wilson (1992). Equilibrium in preemption games with complete information. In M. Majumdar (Ed.), Equilibrium and Dynamics: Essays in Honour of David Gale, pp. 123-147. Basingstoke, Hampshire: Macmillan.

Laraki, R., E. Solan, and N. Vieille (2005). Continuous-time games of timing. J. Econ. Theory 120, 206-238.

Reinganum, J. F. (1981). On the diffusion of new technology: A game theoretic approach. Rev. Econ. Stud. 48(3), 395-405.

Simon, L. K. and M. B. Stinchcombe (1989). Extensive form games in continuous time: Pure strategies. Econometrica 57(5), 1171-1214.

Weeds, H. (2002). Strategic delay in a real options model of R\&D competition. Rev. Econ. Stud. 69, 729-747. 